\title{
Complexity in the signaling network: insights from the use of targeted inhibitors in cancer therapy
}

\author{
Jeremy S. Logue and Deborah K. Morrison ${ }^{1}$ \\ Laboratory of Cell and Developmental Signaling, National Cancer Institute at Frederick, Frederick, Maryland 21702, USA
}

Cancer often arises when normal cellular growth goes awry due to defects in critical signal transduction pathways. A growing number of inhibitors that target specific components of these pathways are in clinical use, but the success of these agents has been limited by the resistance to inhibitor therapy that ultimately develops. Studies have now shown that cancer cells respond to chronic drug treatment by adapting their signaling circuitry, taking advantage of pathway redundancy and routes of feedback and cross-talk to maintain their function. This review focuses on the compensatory signaling mechanisms highlighted by the use of targeted inhibitors in cancer therapy.

Cells respond to a diverse array of signal inputs that control many vital cellular processes, including proliferation, differentiation, migration, and survival. Transmission of the signals that regulate these events occurs through an elaborate network of effector pathways that work together to bring about changes in gene expression, protein function, and cytoskeletal organization required for a particular response. Given that many of these important cellular processes are routinely co-opted in human cancer, it is not surprising that the underlying effector pathways are often dysregulated in tumor cells. Moreover, it has been well established that somatic alterations in key components of these effector pathways can function as driver mutations during oncogenesis. A number of the cancer drivers as well as other core proteins in the pathways have kinase activity, and as a result, they have received much attention from the pharmaceutical industry as "drugable" targets for tumor therapy. The validation that a targeted kinase inhibitor could effectively treat human cancer came with the use of Gleevac (imatinib) to inhibit the Bcr-ABL tyrosine kinase in chronic myelogenous leukemia (CML) (Druker et al. 2001). However, despite the initial success of Gleevec in chronic phase CML patients, it soon became clear that, as with conventional

[Keywords: cancer therapy; signal transduction; targeted inhibitors] ${ }^{1}$ Corresponding author.

E-mail morrisod@mail.nih.gov.

Article is online at http://www.genesdev.org/cgi/doi/10.1101/gad.186965.112. chemotheraputic agents, drug resistance would be a significant problem in targeted inhibitor therapy.

Resistance to inhibitor therapy can be divided into two categories: de novo and acquired (for review, see Janne et al. 2009). De novo resistance refers to the situation in which a drug with proven efficacy fails to elicit any detectable response from the tumor upon initial treatment. In acquired resistance, tumor cells that were initially sensitive to the inhibitor stop responding despite continued administration of the drug. Importantly, it is now known that similar mechanisms can underlie both forms of resistance and that when tumor cells do not respond to drug treatment, it is often due to mutation, loss, or up-regulation of another key signaling protein or pathway. To determine which tumors might exhibit de novo resistance, genetic profiling of the tumor is required to assess whether the tumor possesses an addiction to the protein or pathway that the inhibitor targets and whether the tumor has other genetic alterations that might confer resistance. To identify mechanisms mediating acquired resistance, approaches that have proved most successful include characterization of drug-resistant tumors from patients and the analysis of resistant cancer cell lines that emerge from the continuous culture of drug-sensitive cells with an inhibitor. These strategies have revealed several molecular mechanisms of acquired drug resistance, one of which involves mutation of the target kinase such that drug binding is disrupted. For example, mutation of the gatekeeper residue in the protein kinase domain is a common mechanism conferring resistance to numerous ATPcompetitive tyrosine kinase inhibitors (TKIs) (Carter et al. 2005). In addition to mutation of the target kinase, signaling redundancy as well as alterations in pathway cross-talk and feedback inhibition have emerged as important contributors to drug resistance (Janne et al. 2009).

Cross-talk between effector pathways and feedback inhibition are vital aspects of normal signal transduction that allow cellular responses to be dynamic and adaptive (Mendoza et al. 2011). However, when signaling is blocked by inhibitor treatment, these regulatory loops are often disrupted, causing up-regulation of pathway components and/or activation of parallel circuits. Further complicating the issue, the cross-talk and feedback loops 
can exist at multiple levels of the pathway, and different signaling circuits can be altered or activated, depending on where in the pathway the therapeutic intervention occurs. With regard to signaling redundancy, other members of a protein family or distinct signaling molecules can compensate for the inhibited component to maintain the activity of key downstream circuits even in the presence of drugs. Thus, acquired resistance to inhibitor therapy is not unlike the "Whac-A-Mole" game, where each time the mole is beaten down, he or one of his cohorts pops up again at a different site. In the case of cancer treatment, after the initial oncogene addiction is suppressed by the inhibitor, cells evolve and develop drug resistance to maintain their function, taking advantage of the diversity in the signaling network. In this review, we focus on specific signaling circuits that have been highlighted by the study of inhibitor resistance. We describe the molecular mechanisms that mediate signaling redundancy, pathway cross-talk, and feedback inhibition and discuss the implications of these compensatory signaling mechanisms in cancer therapy.

\section{Receptor tyrosine kinase (RTK) inhibitor resistance: pathway redundancy and cellular reprogramming in ErbB-driven cancers}

RTKs serve as an entry portal for many extracellular cues, and in normal cell signaling, they play a critical role in recruiting the intracellular effector pathways that orchestrate a particular response (Fig. 1). Up-regulated RTK signaling, due to mutation or amplification of the RTK, contributes to a variety of human malignancies, with members of the ErbB family being prominent cancer drivers. The ErbB family consists of four members (ErbB1/ Her1/EGFR, ErbB2/Her2, ErbB3/Her3, and ErbB4/Her4), which form active homo- and heterodimers upon ligand addition (Wieduwilt and Moasser 2008). ErbB2 is unique among the ErbB receptors in that it does not bind a ligand directly, but is preferentially recruited as a binding partner into heterodimers. In contrast, ErbB3 does bind ligands, but is severely compromised in its catalytic activity and cannot phosphorylate exogenous substrates (Shi et al. 2010). Of the ErbB receptors, activating mutations in EGFR are found in $25 \%$ of non-small cell lung carcinomas (NSCLC), and ErbB2 amplification occurs in $20 \%$ of metastatic breast cancer (Wieduwilt and Moasser 2008). Antibody inhibitors that bind regions of the extracellular domain (cetuximab, EGFR; trastuzumab, ErbB2) and ATP-competitive TKIs (erlotinib, gefitnib, and lapatinib) are in clinical use to inhibit ErbB signaling in these cancers. Although gatekeeper mutations are a primary cause of acquired resistance to the TKIs (Pao et al. 2005; Sequist et al. 2011), pathway redundancy has emerged as an important mechanism of resistance for both classes of ErbB inhibitors (Fig. 1; Janne et al. 2009; Garrett and Arteaga 2011). In NSCLC, increased signaling through the MET receptor (typically through MET amplification) can mediate de novo and acquired resistance to
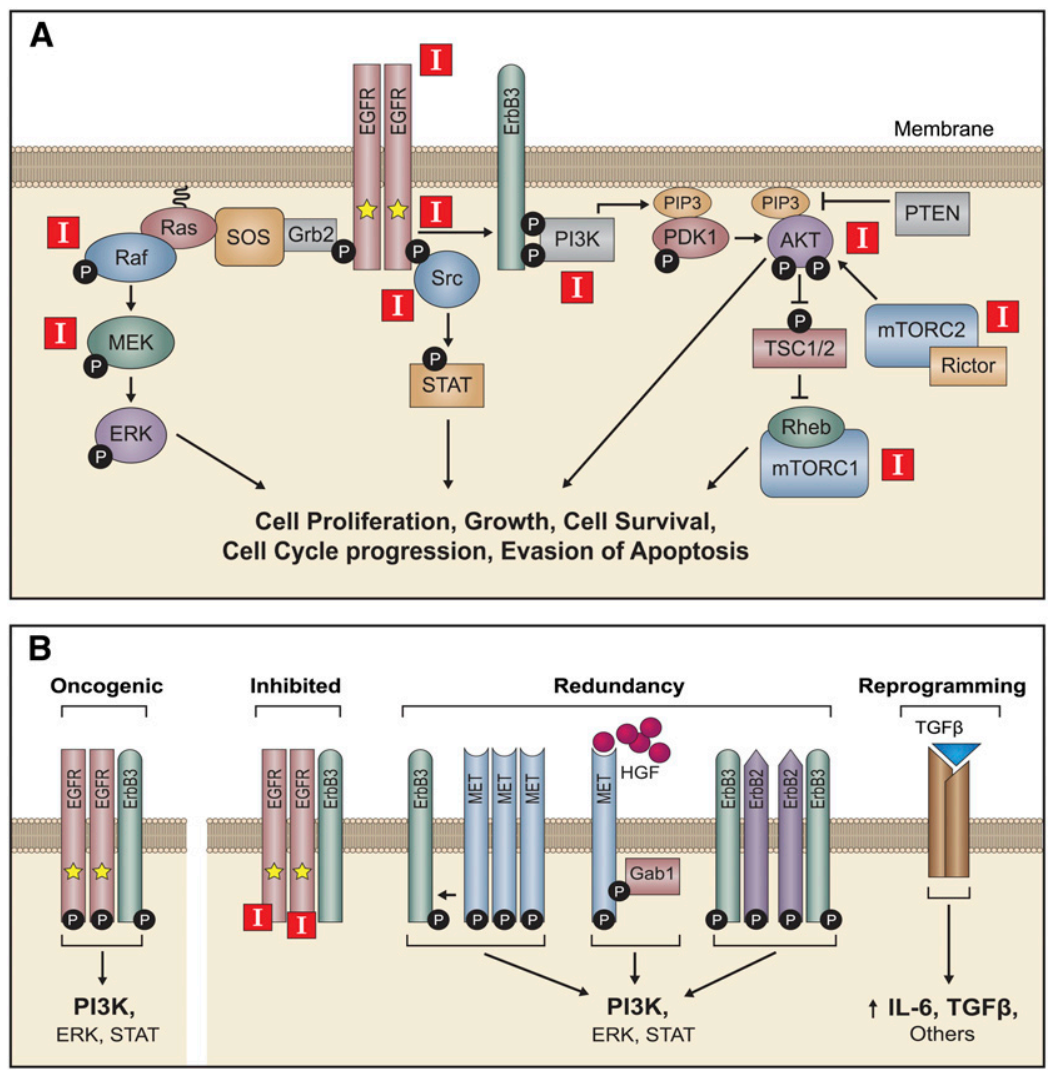

Figure 1. Oncogenic EGFR signaling. $(A) \mathrm{Mu}-$ tant EGFRs engage the Raf/MEK/ERK, PI3K/ $\mathrm{AKT} / \mathrm{mTOR}$, and Src/STAT effector pathways to promote cell proliferation, cell survival, and tumor growth. Proteins targeted by inhibitor (I) therapy are indicated. (B) Signaling redundancy and cellular reprogramming can confer EGFR inhibitor resistance. In the case of signaling redundancy, increased signaling through other RTKs that activate the PI3K, ERK, and STAT pathways eliminates the consequence of EGFR inactivation. In cellular reprogramming, autocrine activation of the TGF $\beta$ receptor promotes a different cellular program, which releases the tumor cells from their dependency on EGFR activation. 
EGFR inhibition in patient tumors and cell lines by engaging similar effector cascades that are activated by EGFR, thus eliminating the consequence of EGFR inactivation (Engelman et al. 2007; Guo et al. 2008; Benedettini et al. 2010; Turke et al. 2010).

Like all RTKs, activated ErbB members function as binding platforms to recruit signaling molecules that typically fall into three categories: enzymes whose activity is stimulated by the RTK (e.g., Src, PI3K, PLC $\gamma$, and Shp2); adaptors that possess protein interaction motifs (e.g., Grb2 and Shc); and docking proteins that when phosphorylated by the RTK, can bind additional signaling molecules (e.g., IRS1/2 and Gab1/2). As a result, an activated RTK complex can engage the repertoire of effector pathways that are needed for a specific biological response. In cancers driven by ErbB family members, the Raf/MEK/ ERK, PI3K/AKT/mTOR, and Src/STAT effector pathways are all activated; however, these tumors are most dependent on PI3K/AKT signaling (Sharma and Settleman 2009; Garrett and Arteaga 2011). EGFR and ErbB2 engage the PI3K/AKT pathway by interacting with ErbB3, which contains multiple docking sites for the $\mathrm{p} 85$ subunit of PI3K. In NSCLCs, amplified MET receptors also interact with ErbB3 and can step in to activate PI3K when EGFR is inhibited (Engelman et al. 2007). Not surprisingly, other RTKs that efficiently promote PI3K signaling can also mediate EGFR inhibitor resistance. For example, activation of IGF-1R through the loss of a negative regulator, IGFBP3, has been found to compensate for EGFR inhibition in A431 squamous carcinoma cells (Guix et al. 2008). In NSCLC and colorectal cancer (CRC) lines, increased ErbB2 signaling can confer resistance to the EGFR antibody inhibitor cetuximab and correlates with intrinsic and acquired resistance to cetuximab in CRC patients (Yonesaka et al. 2011). Studies have also revealed that signaling redundancy and drug resistance can be bidirectional. Not only does amplified MET signaling cause resistance to EGFR inhibitors, but activation of EGFR signaling can compensate for MET inhibition in gastric carcinoma cells (Qi et al. 2010). In breast cancer cells, EGFR activation can mediate resistance to ErbB2 antibody inhibitors (Zhang et al. 2011), and as mentioned above, activation of ErbB2 signaling can bypass the block induced by EGFR antibody inhibitors (Yonesaka et al. 2011).

Another mechanism of EGFR inhibitor resistance that has been observed in lung tumors as well as NSCLC lines correlates with a change in cell morphology to a mesenchymal phenotype (Yao et al. 2010; Sequist et al. 2011). Although the cause for this transition has not been investigated in patient tumors, analysis of resistant cells from the NSCLC line H1650 have indicated a non-RTK compensatory mechanism that involves up-regulated TGF $\beta$ and IL-6 signaling (Fig. 1). Using gene expression profiling, Yao et al. (2010) found that many of the genes differentially expressed in the resistant cells, including IL-6, were known targets of TGF $\beta$. Strikingly, the resistant cells were also found to have increased levels of TGF $\beta$ secretion, indicating an autocrine loop. Further work revealed that depletion of TGF $\beta$ could restore drug sensitivity; inhibit IL-6 expression, which promoted cell sur- vival; and revert the cellular morphology back to an epithelial phenotype. Thus, in this case of acquired resistance, the engagement of a different cellular program appears to release the tumor cells from their dependency on EGFR activation. Moreover, given that inflammation has been shown to augment the expression of both IL- 6 and TGF $\beta$, these findings have important implications for the role of the tumor microenvironment in the development of EGFR inhibitor resistance.

\section{Src enters the fray as an important signaling node}

Src family kinases (SFKs) are overexpressed in many cancer types and are typically associated with advanced malignancies and metastatic spread (Kim et al. 2009). SFKs contribute to cancer progression in a variety of ways, including effects on cell proliferation, migration, and invasion. Recent studies examining the mechanisms of resistance to ErbB2 inhibitor therapy in breast cancer have also implicated the SFKs as an important signaling node that lies downstream from multiple resistance mechanisms (Fig. 2). In particular, SFKs have been shown to be hyperactivated in breast cancer lines whose resistance is associated with the increased expression of certain RTKs, including ErbB2 itself, EGFR, and IGF1R (Rexer et al. 2011; Zhang et al. 2011), as well as cells that mediate an alternative survival pathway through $\beta 1$ integrin (Huang et al. 2011). Moreover, SFKs may help drive resistance in cells that overexpress RTKs by initiating a positive feedback loop. When SFKs interact with activated RTKs, a conformational change occurs in the SFKs that promotes their activation. Activated SFKs are then able to phosphorylate a diverse spectrum of substrates, some of which are the RTKs themselves. In the case of EGFR and ErbB2, phosphorylation by SFKs facilitates receptor dimerization and interactions with ErbB3, thus up-regulating ErbB family signaling (Wheeler et al. 2009; Rexer et al. 2011; Zhang et al. 2011).

Investigation of SFK function in breast cancer signaling has also brought the regulatory relationship between SFKs and the PTEN tumor suppressor into sharper focus (Fig. 2). Loss of PTEN expression is observed in many malignant tumors and is often a marker for advanced neoplastic disease (Li and Ross 2007). PTEN regulates PI3K/AKT signaling through its lipid phosphatase activity, which antagonizes PI3K function by converting PIP3 to PIP2. Reports have identified PTEN as a direct and indirect downstream target of SFKs, with SFKs inducing phosphorylation events that inhibit the membrane recruitment and function of PTEN (Lu et al. 2003; Liang et al. 2010). As a result, in breast cancer cells that express PTEN, preventing Src activation can contribute to the clinical benefit of ErbB2 inhibition by relieving the repression on PTEN (Nagata et al. 2004). It should be noted, however, that this beneficial effect may be abrogated when erythropoietin (EPO) is combined with ErbB2 therapies to combat treatment-related anemia. Although EPO is a cytokine that stimulates erythrocyte production in the bone marrow, its receptor (EpoR) is also expressed in a significant number of primary tumors and breast cancer 

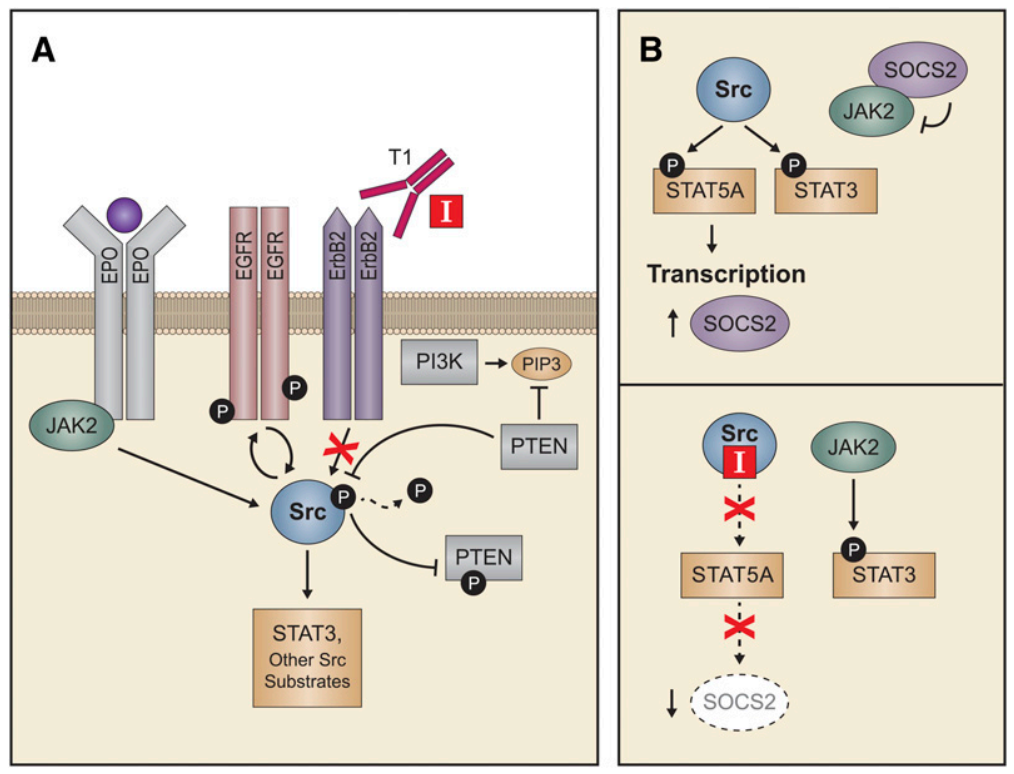

Figure 2. Regulatory circuits involving Src/SFKs. (A) SFKs are hyperactivated in multiple mechanisms of ErbB2 inhibitor (T1, trastuzamab) resistance and can mediate a positive feedback loop promoting RTK dimerization and signaling. Ligand engagement of the EPO receptor also activates Src/SFKs via the JAK2 kinase. In addition, the reciprocal regulatory relationship between SFKs and PTEN is indicated. Dephosphorylation of SFKs by PTEN's protein phosphatase activity inactivates the SFKs, whereas phosphorylation of PTEN induced by SFKs inhibits the function of PTEN. (B) JAK2 reactivates STAT3 in response to SFK inhibition via a signaling circuit involving the STAT5A and SOCS2 proteins.

lines that are ErbB2-positive (Liang et al. 2010). In cells that coexpress EpoR and ErbB2, EpoR activation has been found to reduce the effectiveness of trastuzamab by promoting Src activation (via the JAK2 kinase) and, in turn, PTEN phosphorylation and inactivation (Liang et al. 2010).

In a reciprocal manner, PTEN has also been implicated as a negative regulator of the SFKs. PTEN is well known for its lipid phosphatase activity; however, it was first characterized as a dual specificity protein phosphatase (Myers et al. 1997), and it is the protein phosphatase activity of PTEN that has been attributed to SFK regulation. Using PTEN-deficient cells reconstituted with wildtype or mutant PTEN proteins, Zhang et al. (2011) found that PTEN could modulate activated Src levels in breast cancer lines and that this ability correlated solely with the protein phosphatase activity of PTEN. Similar results have been observed in studies examining PTEN function in glioma cell migration (Dey et al. 2008). Moreover, the work of Dey et al. (2008) indicates that loss of PTEN, which occurs in $>60 \%$ of gliomas, may in part promote integrindirected cell migration through up-regulated SFK signaling.

SFKs have important functions with regard to cell motility; however, they also promote cell proliferation and survival by activating members of the STAT transcription factors, chiefly STAT3. In cancer cells, SFK inhibitors dramatically reduce cell migration and invasion, but have only a modest effect on cell survival (Johnson et al. 2005). Interestingly, activated STAT3 has been identified in preclinical studies as a potential biomarker for SFK inhibitor resistance, suggesting a compensatory circuit for STAT3 activation (Johnson et al. 2007). Insight as to how STAT3 might be reactivated in Srcinhibited cells comes from work conducted by the laboratory of Dr. Faye Johnson (Byers et al. 2009; Sen et al. 2009, 2012). In initial studies using NSCLC and head and neck squamous cell carcinoma (HNSCC) lines, sustained SFK inhibition was shown to cause only a transient inhibition of STAT3, with reactivation dependent on JAK2 kinase activity (Byers et al. 2009; Sen et al. 2009). Subsequent work revealed that the JAK2-dependent reactivation of STAT3 involves the SOCS2 protein and another STAT family member, STAT5 (Sen et al. 2012). In contrast to STAT3, the inhibition of STAT5 by SFK TKIs was lasting, resulting in reduced expression of SOCS2 via STAT5A inhibition. SOCS2 is a known inhibitor of JAK/STAT binding (Rico-Bautista et al. 2006), and the overexpression of SOCS2 was shown to prevent STAT3 reactivation and enhance the cytotoxic effects of SFK inhibitor treatment (Sen et al. 2012). In this signaling circuit, SFK inhibition leads to diminished SOCS2 expression via sustained STAT5A inhibition, thus allowing JAK2/STAT3 binding and reactivation of STAT3 (Fig. 2B).

\section{Blockade of PI3K/AKT/mTOR signaling: effects on pathway cross-talk and feedback inhibition}

The PI3K/AKT/mTOR effector pathway is one of the most dysregulated signaling cascades in human cancer (Yuan and Cantley 2008). The pathway is typically "ON" in human tumors due to activation by oncogenic RTK and Ras proteins, loss of PTEN, or mutational activation of key pathway components, such as PI3KCA or AKT. Under normal signaling conditions, PI3K is recruited to the cell surface through binding to either an RTK, an RTKassociated protein, or GTP-bound Ras. At the membrane, PI3K phosphorylates PIP2 to generate PIP3, a critical second messenger that is needed for the subsequent membrane localization of the downstream kinases PDK1 and AKT (Cantley 2002). AKT is well known for its role in promoting cell survival and is activated at the cell surface through phosphorylation events mediated by PDK1 and mTORC2. mTORC2 and mTORC1 are multiprotein kinase complexes, with mTOR functioning as the catalytic component in each. Interestingly, while AKT is a down- 
stream substrate of mTORC2, it also functions as an upstream regulator of $\mathrm{mTORC1}$, a central signaling node that integrates growth signals with nutrient and energy supplies (Huang and Manning 2009; Zoncu et al. 2011). In growth factor signaling, AKT promotes mTORC1 activation by phosphorylating the TSC1/TSC2 tumor suppressor complex and blocking its inhibitory effect on the RhebGTPase, which is an activating subunit of mTORC1. Activated mTORCl drives protein synthesis, and two of its key substrates are S6K and 4E-BP1 (Burnett et al. 1998).

Early studies characterizing mTORC1 were aided by the use of rapamycin, a natural product inhibitor that targets the mTORC1 complex but has little effect on mTORC2 (Sabatini et al. 1994). Strikingly, the continued use of rapamycin and its derivatives (rapalogs) in cell culture systems and cancer treatment has highlighted the existence of numerous mTORC1-dependent feedback circuits that when unleashed, can impact drug resistance (Fig. 3). The first and best-characterized of these feedback loops impinges on the IRS1 docking protein and is mediated by mTORC1-activated S6K. Biochemical studies have shown that active S6K down-regulates the expression of IRS1 through poorly understood transcriptional mechanisms and can block IRS1 function by direct phosphorylation (Harrington et al. 2004; Shah et al. 2004). Specifically, phosphorylation of IRS1 by S6K inhibits binding to the insulin receptor (IR) and IGF-1R and promotes IRS1 degradation. mTORC1 inhibition severs this feedback loop, and elevated AKT activation that correlates with increased IRS1 levels has been observed in breast cancer lines and patient tumors treated with rapamycin or rapalogs (O'Reilly et al. 2006).

Inactivation of $\mathrm{S} 6 \mathrm{~K}$ through mTORC1 inhibition has also been found to promote PI3K/AKT activity through IRS1-independent mechanisms, one of which involves up-regulated RTK signaling that promotes PI3K activation. Through relief in S6K transcriptional effects, increased expression of PDGFR in mouse embryo fibroblasts (Zhang et al. 2007), up-regulation of IGF-1R in rhabdomyosarcoma lines (Wan et al. 2007), and activation of an IGF-1/IGF-1R autocrine loop due to the increased expression of IGF1 and IRS2 in AML cells (Tamburini et al. 2008) have all been reported to activate AKT in rapamycintreated cells. Another feedback mechanism mediated by S6K involves phosphorylation of Rictor, a protein specific to the mTORC2 complex. Three independent studies have found that S6K phosphorylates Rictor on T1135 in a rapamycin-sensitive manner and that this phosphorylation represses the function of $\mathrm{mTORC} 2$ as an AKT activator (Dibble et al. 2009; Julien et al. 2010; Treins et al. 2010). Phosphorylation of this site did not inhibit mTORC2 assembly or catalytic activity, but was found to mediate binding of 14-3-3 proteins, suggesting that sequestration of the complex may be involved.

In addition to feedback inhibition mediated by S6K, two key studies published in 2011 report another feedback mechanism that is the direct result of mTORC1 activity (Hsu et al. 2011; Yu et al. 2011). In both studies, a phosphoproteomic screen was conducted to uncover new substrates of the mTOR complexes, and Grb10 was identified as a specific mTORC1 substrate. Grb10 belongs to a small family of adaptor proteins that interacts with activated IR and IGF-1R. Binding of Grb10 suppresses the intrinsic kinase activity of these receptors, which in turn blocks the phosphorylation of key downstream substrates (Stein et al. 2001). mTORC1-dependent phosphorylation of Grb10 was shown by both groups to prevent Grb10 degradation, thus increasing Grb10 levels and its inhibitory potential. Notably, analysis of tumor microarray data indicates that mRNA expression of Grb10 is reduced in

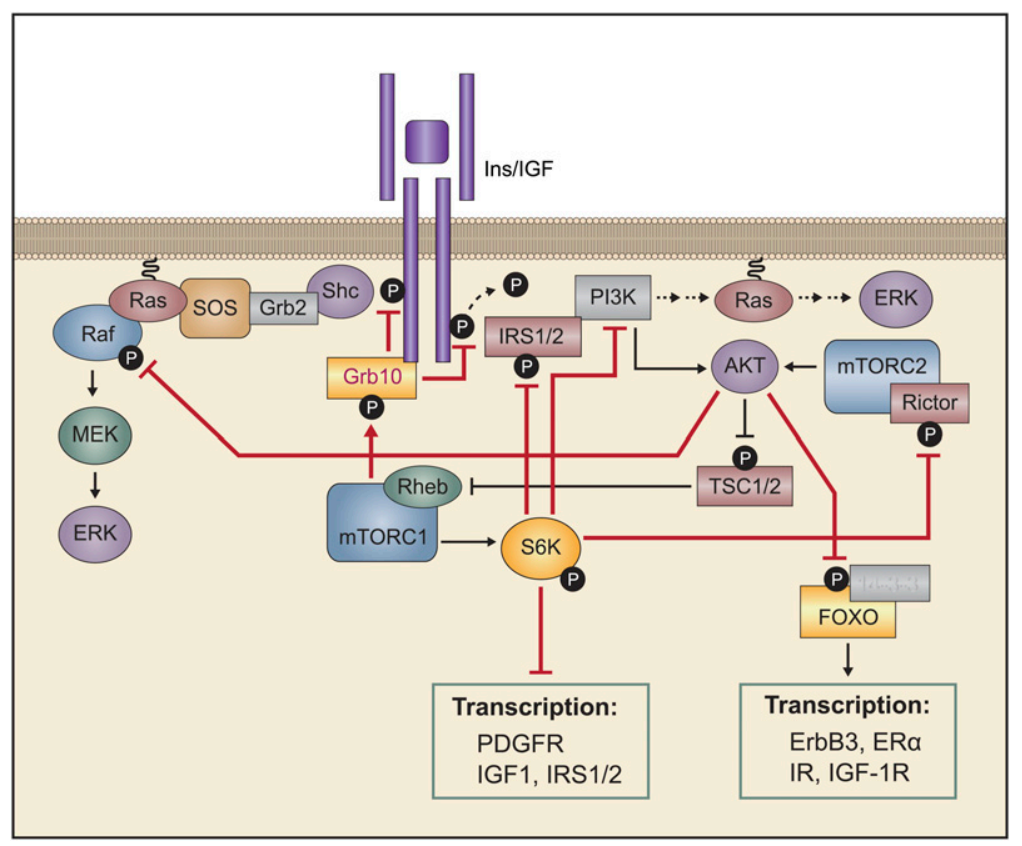

Figure 3. Feedback inhibition mediated by the PI3K/AKT/mTOR pathway. Pathway cross-talk and inhibitory feedback circuits induced by activated components of the PI3K/AKT/mTOR pathway are shown. Pathway connections that represent mechanisms of negative feedback are denoted by a red line. Disruption of the feedback circuits by inhibitor treatment can alter the effectiveness of drug therapy. Transcriptional events that occur downstream from S6K and FOXO are also indicated. 
a variety of human cancers, suggesting that Grb10 may have tumor suppressor activity (Hsu et al. 2011; Yu et al. 2011).

Feedback loops emanating from mTORC1 can also inhibit signaling in the parallel ERK pathway. In the studies described above, depletion of Grb10 was found to increase ERK activation as well as PI3K/AKT signaling (Hsu et al. 2011; Yu et al. 2011). This effect is likely due to a relief in the Grb10 block on IR and IGF-1R, which also affects signal transmission to the ERK effector cascade. In addition, elevated ERK activation has been observed in tumors of metastatic cancer patients treated with the rapalog Rad-001. Using cell culture systems to further elucidate this effect, Carracedo et al. (2008) found that the hyperactivation of ERK required Ras, PI3K, and MEK function, but not mTORC2 or AKT activity, and could be abrogated by a constitutively active S6K. These findings indicate an S6K-mediated brake on PI3K, whose relief promotes Ras-dependent ERK activation. Although ERK activation could be suppressed by PI3K inhibitors, the mechanism for how S6K mediates a brake on PI3K or how PI3K promotes Ras to ERK signaling remains unclear.

PI3K/AKT signaling affects various cellular processes in addition to mTORCl activation, and it is not surprising that drugs that target upstream components in the pathway have exposed mTORC1-independent feedback circuits that can alter inhibitor therapy (Fig. 3). One such circuit involves the FOXO transcription factors, known substrates of the AKT kinase. Under normal signaling conditions, phosphorylation of the FOXOs by AKT promotes 14-3-3 binding, which sequesters these transcription factors in the cytosol (Tzivion et al. 2011). Inhibitors that suppress AKT signaling can promote the nuclear localization of FOXO, thus inducing FOXO-dependent transcriptional changes that increase the expression of certain signaling receptors. In ErbB2-expressing breast cancer cells, inhibition of PI3K or AKT, but not mTORC1, has been found to promote the expression of ErbB3, causing increased ErbB2/ErbB3 signaling and compensatory activation of the ERK cascade (Serra et al. 2011). Further studies have revealed that AKT inhibition induces ErbB3, IR, and IGF-1R mRNA and protein levels in various cancer lines and that this effect can be largely abrogated by FOXO depletion (Chandarlapaty et al. 2011). The FOXO proteins appear to be directly responsible for the induced transcription of the receptor genes and could be coimmunoprecipitated with their $5^{\prime}$ untranslated regions (UTRs). Activation of FOXO3a has also been found to up-regulate estrogen receptor (ER) levels and ER signaling in breast tumors and cancer lines that overexpress ErbB2 and are $\mathrm{ER}^{+}$(Guo and Sonenshein 2004). Moreover, in BT474 breast cancer cells, ER signaling induced by AKT inhibition and FOXO3a derepression was able to promote cell survival in the presence of the EGFR/ErbB2 inhibitor lapatinib (Xia et al. 2006). In this and all cases where receptor expression was induced by FOXO activation, combined inhibition of the up-regulated receptor and the PI3K pathway has been shown to restore growth arrest.

\section{Blockade of Raf/MEK/ERK signaling: pathway reactivation and up-regulation of parallel circuits}

Another effector pathway that is up-regulated in a high percentage of human tumors is the ERK cascade, comprised of the Raf, MEK, and ERK kinases (for review, see Shaul and Seger 2007). These kinases are activated in a sequential manner through direct phosphorylation, and a primary function of ERK signaling is to promote cell cycle progression and cell proliferation. Under normal conditions, engagement of the ERK cascade begins when members of the Raf family interact directly with activated Ras. Binding to Ras recruits the cytosolic Raf proteins to the plasma membrane, disrupts their autoinhibited state, and promotes their activation through the formation of Raf homo- and heterodimers. Once activated, the Raf kinases phosphorylate and activate MEK1/2, which in turn phosphorylates and activates ERK1/2.

In human cancer, ERK signaling is driven by oncogenic Ras and RTK proteins, as well as by mutationally activated B-Raf proteins. Oncogenic B-Raf mutations generally bypass the need for Ras binding and promote constitutive Raf activation by destabilizing the inactive kinase conformation (Wan et al. 2004). Although numerous B-Raf mutations have been identified in human cancer, alterations affecting Val 600 predominate and cause high catalytic activity. The observation that the V600E-B-Raf mutant is found in $\sim 50 \%$ of malignant melanomas as well as many colorectal and thyroid cancers has hastened the development of a variety of ATP-competitive Raf inhibitors, some of which have high specificity toward V600E-BRaf. Strikingly, the use of these drugs has revealed one of the most unexpected consequences of targeted inhibitor therapy in that they all paradoxically promote Raf signaling under conditions where Raf is activated via dimer formation (Hatzivassiliou et al. 2010; Heidorn et al. 2010; Poulikakos et al. 2010). More specifically, when the Rafs localize with Ras at the membrane, binding of the inhibitor to one member of the Raf dimer appears to stabilize dimer formation and induce a conformational change that promotes the activation of the second dimer member. Thus, while these inhibitors effectively block ERK activation driven by V600E-B-Raf, they stimulate ERK signaling in the presence of oncogenic or normally activated Ras proteins. Further complicating the issue, some of the Raf inhibitors also promote binding between the B-Raf kinase and members of the closely related KSR scaffold family (Hu et al. 2011; McKay et al. 2011). In these cases, high expression of KSR can moderate the effect of inhibitor treatment by restricting B-Raf's ability to dimerize with other Raf proteins.

This complexity in inhibitor effects is the proverbial two-edged sword, having both advantages and disadvantages. The primary advantage is that when used to treat tumors driven by V600E-B-Raf, these inhibitors specifically suppress ERK activation in tumor cells but not in normal cells, thus reducing toxicity. However, a major disadvantage is the demonstrated ability of the Raf inhibitors to facilitate secondary tumor formation (Lacouture et al. 2012). Approximately $15 \%-30 \%$ of patients treated 
with these drugs develop skin keratoacanthomas and/or squamous cell carcinomas that can harbor H-Ras mutations (Su et al. 2012b). Although these tumors are easily detected and removed, the potential for tumor progression in internal tissues that possess dormant Ras mutations raises significant concern for the use of these inhibitors in long-term or adjuvant therapies. Also of concern is that paradoxical Raf activation can promote resistance to the Raf inhibitors, with perturbations that induce Raf dimerization detected in resistant tumors and cell lines (Solit and Rosen 2011). Interestingly, paradoxical Raf activation can also influence responses to the Bcr-ABL inhibitors imatinib, nilotinib, and dasatinib that have weak off-target activity against Raf. These inhibitors have been recently shown to promote Ras-dependent Raf dimerization, and when drug-resistant Bcr-ABL mutants emerge, the reactivation of Ras drives the paradoxical activation of Raf, thus increasing the dependency of the resistant CML cells on ERK signaling (Packer et al. 2011).

To date, the mechanisms of Raf inhibitor resistance have been evaluated mainly in the context of melanoma patients and various cancer cell lines that express V600EB-Raf. Somewhat unexpectedly, secondary mutations that disrupt inhibitor binding have not been observed, even though mutation of the B-Raf gatekeeper site is known to prevent drug binding (Whittaker et al. 2010). As indicated above, several of the identified mechanisms of resistance have the consequence of promoting the dimerization of wild-type Raf proteins in drug-treated cells (Fig. 4). These mechanisms include activating mutations in N-Ras or K-Ras (Nazarian et al. 2010; Su et al. 2012a),

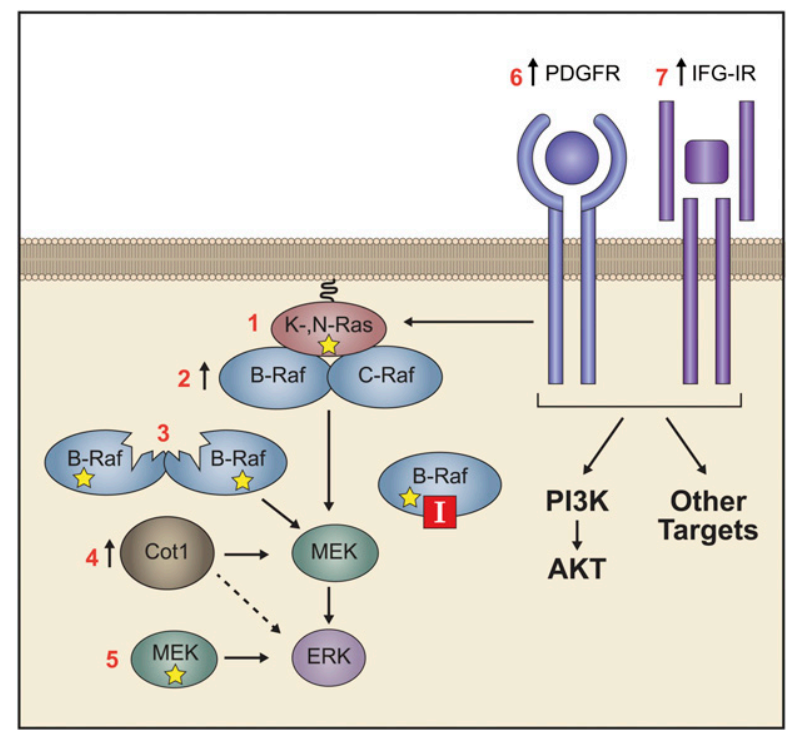

Figure 4. Mechanisms of Raf inhibitor resistance. Raf inhibitor resistance can occur through mechanisms that reactivate ERK signaling, including activating Ras mutations (1), increased Raf expression (2), truncation of V600E-B-Raf (3), increased Cot1 expression (4), and activating MEK mutation (5). Activation of compensatory pathways, such as up-regulated PDGFR (6) and IGF-1R signaling (7), can also confer Raf inhibitor resistance. increased expression of B-Raf or C-Raf (Corcoran et al. 2010; Johannessen et al. 2010), and increased signaling through RTKs that promote Ras activation (Nazarian et al. 2010; Villanueva et al. 2010). In addition, a splice variant of V600E-B-Raf that has enhanced dimerization potential has recently been identified in melanoma lines resistant to the Raf inhibitor vemurafenib (Poulikakos et al. 2011). These truncated proteins lack the Ras-binding domain and dimerize in a Ras-independent manner. Dimerization of these truncated V600E mutants was found to be critical for inhibitor resistance, as mutation of their dimer interface restored drug sensitivity. Strikingly, V600E B-Raf splice variants that lack the Ras-binding domain have been observed in a significant number of relapsed melanoma patients, and these transcripts were not detected prior to treatment (Poulikakos et al. 2011).

The pertubations that promote Raf dimerization are known to reactivate ERK signaling in drug-treated cells, and in general, acquired resistance to Raf inhibitors can be divided into two categories: one that is dependent on ERK reactivation and another that involves the up-regulation of alternative pathways that reduce ERK dependency (Fig. 4). Additional examples of the first category include mutational activation of MEK1 (C121S) (Wagle et al. 2011) and increased expression of the Cot1 kinase, which directly activates MEK (Johannessen et al. 2010). Examples of the second category include activating mutations in AKT3 (Shao and Aplin 2010) and up-regulated signaling from certain RTKs, including the PDGFR and the IGF1R (Nazarian et al. 2010; Villanueva et al. 2010). Although these receptors can activate the ERK cascade, they strongly promote PI3K/AKT signaling, and increased AKT activation has been observed in resistant tumors and cell lines with up-regulated PDGFR or IGF1R signaling. Notably, in many of the cases where Raf inhibitor resistance is mediated by ERK reactivation, such as in cells expressing activated Ras proteins or truncated V600E-B-Raf mutants, the addition of MEK inhibitors has been found to restore growth arrest (Nazarian et al. 2010; Villanueva et al. 2010; Poulikakos et al. 2011). In contrast, when upregulation of PI3K/AKT signaling is observed, the addition of MEK inhibitors has little effect, and combined inhibition of both the PI3K and ERK pathways is required for a cytotoxic response (Shi et al. 2011).

Drugs targeting the MEK kinases have also been used in the clinical setting. Although MEK mutations are rare in human cancer, MEK is often up-regulated in tumors due to mutational activation of upstream pathway components. Recent studies examining colon cancer lines resistant to the allosteric MEK inhibitor AZD6244 have found that an activating MEK1 mutation (F12L) or upregulation of driver oncogenes (G13D-K-Ras or V600EB-Raf) can mediate drug resistance. In particular, when the driver oncogene was G13D-K-Ras, K-Ras amplification was found to reactivate multiple Ras effector pathways. However, when V600E-B-Raf was the amplified driver, only ERK signaling was reactivated, and cotreatment with MEK and Raf inhibitors could restore growth arrest. These findings further support the model that in tumors with a high dependency on ERK signaling, the combination of 
Raf and MEK inhibitors may have increased clinical efficacy than either therapy alone.

\section{Concluding remarks}

Cancer treatment continues to evolve from the sledgehammer approach of agents that kill diseased and healthy cells indiscriminately to the silver bullet approach of targeted therapeutics that selectively inhibit a critical signaling component in the tumorigenic pathway. To date, however, these silver bullets have been unable to deliver a fatal blow to a human cancer and have only provided a temporary reprieve due to the development of drug resistance. Elucidating the mechanisms of inhibitor resistance has emphasized the need to confront a complicated disease such as cancer from a signaling network perspective, taking into account how effector pathways interconnect and adapt. In addition, these studies have demonstrated the importance of understanding the detailed regulatory mechanisms involved in signal transduction. Thus, in this new era of personalized cancer therapy, decades of research spent studying signaling pathways through genetic and biochemical means is proving to be highly valuable in helping to understand tumor profiles, establish biomarkers, and guide choices for combinatorial drug therapies. In particular, studies investigating the mechanisms of resistance have established that combined inhibition of the driver oncoprotein and key pathways or proteins commonly activated in drug resistance should prove beneficial. Moreover, it is now clear that patient profiling will be critical for determining the first and subsequent lines of combinatorial treatment and that monitoring of tumors at the signaling level during treatment will be required to achieve a durable response. The ultimate hope is that some combination of silver bullets will impose a series of signaling blocks that cannot be bypassed, thus delivering a mortal wound that eradicates the cancer.

\section{Acknowledgments}

Our work is supported by federal funds from the National Cancer Institute.

\section{References}

Benedettini E, Sholl LM, Peyton M, Reilly J, Ware C, Davis L, Vena N, Bailey D, Yeap BY, Fiorentino M, et al. 2010. Met activation in non-small cell lung cancer is associated with de novo resistance to EGFR inhibitors and the development of brain metastasis. Am J Pathol 177: 415-423.

Burnett PE, Barrow RK, Cohen NA, Snyder SH, Sabatini DM. 1998. RAFT1 phosphorylation of the translational regulators p70 S6 kinase and 4E-BP1. Proc Natl Acad Sci 95: 1432-1437.

Byers LA, Sen B, Saigal B, Diao L, Wang J, Nanjundan M, Cascone T, Mills GB, Heymach JV, Johnson FM. 2009. Reciprocal regulation of c-Src and STAT3 in non-small cell lung cancer. Clin Cancer Res 15: 6852-6861.

Cantley LC. 2002. The phosphoinositide 3-kinase pathway. Science 296: 1655-1657.

Carracedo A, Ma L, Teruya-Feldstein J, Rojo F, Salmena L, Alimonti A, Egia A, Sasaki AT, Thomas G, Kozma SC, et al.
2008. Inhibition of mTORC1 leads to MAPK pathway activation through a PI3K-dependent feedback loop in human cancer. J Clin Invest 118: 3065-3074.

Carter TA, Wodicka LM, Shah NP, Velasco AM, Fabian MA, Treiber DK, Milanov ZV, Atteridge CE, Biggs WH 3rd, Edeen PT, et al. 2005. Inhibition of drug-resistant mutants of ABL, KIT, and EGF receptor kinases. Proc Natl Acad Sci 102: 11011-11016.

Chandarlapaty S, Sawai A, Scaltriti M, Rodrik-Outmezguine V, Grbovic-Huezo O, Serra V, Majumder PK, Baselga J, Rosen N. 2011. AKT inhibition relieves feedback suppression of receptor tyrosine kinase expression and activity. Cancer Cell 19: $58-71$.

Corcoran RB, Dias-Santagata D, Bergethon K, Iafrate AJ, Settleman J, Engelman JA. 2010. BRAF gene amplification can promote acquired resistance to MEK inhibitors in cancer cells harboring the BRAF V600E mutation. Sci Signal 3: ra84. doi: 10.1126/ scisignal.2001148.

Dey N, Crosswell HE, De P, Parsons R, Peng Q, Su JD, Durden DL. 2008. The protein phosphatase activity of PTEN regulates SRC family kinases and controls glioma migration. Cancer Res 68: 1862-1871.

Dibble CC, Asara JM, Manning BD. 2009. Characterization of Rictor phosphorylation sites reveals direct regulation of mTOR complex 2 by S6K1. Mol Cell Biol 29: 5657-5670.

Druker BJ, Talpaz M, Resta DJ, Peng B, Buchdunger E, Ford JM, Lydon NB, Kantarjian H, Capdeville R, Ohno-Jones S, et al. 2001. Efficacy and safety of a specific inhibitor of the BCRABL tyrosine kinase in chronic myeloid leukemia. $N$ Engl $J$ Med 344: 1031-1037.

Engelman JA, Zejnullahu K, Mitsudomi T, Song Y, Hyland C, Park JO, Lindeman N, Gale CM, Zhao X, Christensen J, et al. 2007. MET amplification leads to gefitinib resistance in lung cancer by activating ERBB3 signaling. Science 316: 1039-1043.

Garrett JT, Arteaga CL. 2011. Resistance to HER2-directed antibodies and tyrosine kinase inhibitors: Mechanisms and clinical implications. Cancer Biol Ther 11: 793-800.

Guix M, Faber AC, Wang SE, Olivares MG, Song Y, Qu S, Rinehart C, Seidel B, Yee D, Arteaga CL, et al. 2008. Acquired resistance to EGFR tyrosine kinase inhibitors in cancer cells is mediated by loss of IGF-binding proteins. I Clin Invest 118: 2609-2619.

Guo S, Sonenshein GE. 2004. Forkhead box transcription factor FOXO3a regulates estrogen receptor $\alpha$ expression and is repressed by the Her-2/neu/phosphatidylinositol 3-kinase/ Akt signaling pathway. Mol Cell Biol 24: 8681-8690.

Guo A, Villen J, Kornhauser J, Lee KA, Stokes MP, Rikova K, Possemato A, Nardone J, Innocenti G, Wetzel R, et al. 2008. Signaling networks assembled by oncogenic EGFR and c-Met. Proc Natl Acad Sci 105: 692-697.

Harrington LS, Findlay GM, Gray A, Tolkacheva T, Wigfield S, Rebholz H, Barnett J, Leslie NR, Cheng S, Shepherd PR, et al. 2004. The TSC1-2 tumor suppressor controls insulin-PI3K signaling via regulation of IRS proteins. J Cell Biol 166: 213223.

Hatzivassiliou G, Song K, Yen I, Brandhuber BJ, Anderson DJ, Alvarado R, Ludlam MJ, Stokoe D, Gloor SL, Vigers G, et al. 2010. RAF inhibitors prime wild-type RAF to activate the MAPK pathway and enhance growth. Nature 464: 431-435.

Heidorn SJ, Milagre C, Whittaker S, Nourry A, Niculescu-Duvas I, Dhomen N, Hussain J, Reis-Filho JS, Springer CJ, Pritchard C, et al. 2010. Kinase-dead BRAF and oncogenic RAS cooperate to drive tumor progression through CRAF. Cell 140: 209-221.

Hsu PP, Kang SA, Rameseder J, Zhang Y, Ottina KA, Lim D, Peterson TR, Choi Y, Gray NS, Yaffe MB, et al. 2011. The 
mTOR-regulated phosphoproteome reveals a mechanism of mTORC1-mediated inhibition of growth factor signaling. Science 332: 1317-1322.

Hu J, Yu H, Kornev AP, Zhao J, Filbert EL, Taylor SS, Shaw AS. 2011. Mutation that blocks ATP binding creates a pseudokinase stabilizing the scaffolding function of kinase suppressor of Ras, CRAF and BRAF. Proc Natl Acad Sci 108: 6067-6072.

Huang J, Manning BD. 2009. A complex interplay between Akt, TSC2 and the two mTOR complexes. Biochem Soc Trans 37: 217-222.

Huang C, Park CC, Hilsenbeck SG, Ward R, Rimawi MF, Wang YC, Shou J, Bissell MJ, Osborne CK, Schiff R. 2011. $\beta 1$ integrin mediates an alternative survival pathway in breast cancer cells resistant to lapatinib. Breast Cancer Res 13: R84. doi: $10.1186 /$ cbr2936.

Janne PA, Gray N, Settleman J. 2009. Factors underlying sensitivity of cancers to small-molecule kinase inhibitors. Nat Rev Drug Discov 8: 709-723.

Johannessen CM, Boehm JS, Kim SY, Thomas SR, Wardwell L, Johnson LA, Emery CM, Stransky N, Cogdill AP, Barretina J, et al. 2010. COT drives resistance to RAF inhibition through MAP kinase pathway reactivation. Nature 468: 968-972.

Johnson FM, Saigal B, Talpaz M, Donato NJ. 2005. Dasatinib (BMS-354825) tyrosine kinase inhibitor suppresses invasion and induces cell cycle arrest and apoptosis of head and neck squamous cell carcinoma and non-small cell lung cancer cells. Clin Cancer Res 11: 6924-6932.

Johnson FM, Saigal B, Tran H, Donato NJ. 2007. Abrogation of signal transducer and activator of transcription 3 reactivation after Src kinase inhibition results in synergistic antitumor effects. Clin Cancer Res 13: 4233-4244.

Julien LA, Carriere A, Moreau J, Roux PP. 2010. mTORC1activated S6K1 phosphorylates Rictor on threonine 1135 and regulates mTORC2 signaling. Mol Cell Biol 30: 908-921.

Kim LC, Song L, Haura EB. 2009. Src kinases as therapeutic targets for cancer. Nat Rev Clin Oncol 6: 587-595.

Lacouture ME, O'Reilly K, Rosen N, Solit DB. 2012. Induction of cutaneous squamous cell carcinomas by RAF inhibitors: Cause for concern? I Clin Oncol 30: 329-330.

Li L, Ross AH. 2007. Why is PTEN an important tumor suppressor? J Cell Biochem 102: 1368-1374.

Liang K, Esteva FJ, Albarracin C, Stemke-Hale K, Lu Y, Bianchini G, Yang CY, Li Y, Li X, Chen CT, et al. 2010. Recombinant human erythropoietin antagonizes trastuzumab treatment of breast cancer cells via Jak2-mediated Src activation and PTEN inactivation. Cancer Cell 18: 423-435.

Lu Y, Yu Q, Liu JH, Zhang J, Wang H, Koul D, McMurray JS, Fang X, Yung WK, Siminovitch KA, et al. 2003. Src family protein-tyrosine kinases alter the function of PTEN to regulate phosphatidylinositol 3-kinase/AKT cascades. J Biol Chem 278: 40057-40066.

McKay MM, Ritt DA, Morrison DK. 2011. RAF inhibitorinduced KSR1/B-RAF binding and its effects on ERK cascade signaling. Curr Biol 21: 563-568.

Mendoza MC, Er EE, Blenis J. 2011. The Ras-ERK and PI3KmTOR pathways: Cross-talk and compensation. Trends Biochem Sci 36: 320-328.

Myers MP, Stolarov JP, Eng C, Li J, Wang SI, Wigler MH, Parsons R, Tonks NK. 1997. P-TEN, the tumor suppressor from human chromosome 10q23, is a dual-specificity phosphatase. Proc Natl Acad Sci 94: 9052-9057.

Nagata Y, Lan KH, Zhou X, Tan M, Esteva FJ, Sahin AA, Klos KS, Li P, Monia BP, Nguyen NT, et al. 2004. PTEN activation contributes to tumor inhibition by trastuzumab, and loss of
PTEN predicts trastuzumab resistance in patients. Cancer Cell 6: 117-127.

Nazarian R, Shi H, Wang Q, Kong X, Koya RC, Lee H, Chen Z, Lee MK, Attar N, Sazegar H, et al. 2010. Melanomas acquire resistance to B-RAF(V600E) inhibition by RTK or N-RAS upregulation. Nature 468: 973-977.

O'Reilly KE, Rojo F, She QB, Solit D, Mills GB, Smith D, Lane H, Hofmann F, Hicklin DJ, Ludwig DL, et al. 2006. mTOR inhibition induces upstream receptor tyrosine kinase signaling and activates Akt. Cancer Res 66: 1500-1508.

Packer LM, Rana S, Hayward R, O'Hare T, Eide CA, Rebocho A, Heidorn S, Zabriskie MS, Niculescu-Duvaz I, Druker BJ, et al. 2011. Nilotinib and MEK inhibitors induce synthetic lethality through paradoxical activation of RAF in drugresistant chronic myeloid leukemia. Cancer Cell 29: 715727.

Pao W, Miller VA, Politi KA, Riely GJ, Somwar R, Zakowski MF, Kris MG, Varmus H. 2005. Acquired resistance of lung adenocarcinomas to gefitinib or erlotinib is associated with a second mutation in the EGFR kinase domain. PLoS Med 2: e73. doi: 10.1371/journal.pmed.0020073.

Poulikakos PI, Zhang C, Bollag G, Shokat KM, Rosen N. 2010. RAF inhibitors transactivate RAF dimers and ERK signalling in cells with wild-type BRAF. Nature 464: 427-430.

Poulikakos PI, Persaud Y, Janakiraman M, Kong X, Ng C, Moriceau G, Shi H, Atefi M, Titz B, Gabay MT, et al. 2011. RAF inhibitor resistance is mediated by dimerization of aberrantly spliced BRAF(V600E). Nature 480: 387-390.

Qi J, McTigue MA, Rogers A, Lifshits E, Christensen JG, Janne PA, Engelman JA. 2010. Multiple mutations and bypass mechanisms can contribute to development of acquired resistance to MET inhibitors. Cancer Res 71: 1081-1091.

Rexer BN, Ham AJ, Rinehart C, Hill S, Granja-Ingram Nde M, Gonzalez-Angulo AM, Mills GB, Dave B, Chang JC, Liebler DC, et al. 2011. Phosphoproteomic mass spectrometry profiling links Src family kinases to escape from HER 2 tyrosine kinase inhibition. Oncogene 30: 4163-4174.

Rico-Bautista E, Flores-Morales A, Fernandez-Perez L. 2006. Suppressor of cytokine signaling (SOCS) 2, a protein with multiple functions. Cytokine Growth Factor Rev 17: 431439.

Sabatini DM, Erdjument-Bromage H, Lui M, Tempst P, Snyder SH. 1994. RAFT1: A mammalian protein that binds to FKBP12 in a rapamycin-dependent fashion and is homologous to yeast TORs. Cell 78: 35-43.

Sen B, Saigal B, Parikh N, Gallick G, Johnson FM. 2009. Sustained Src inhibition results in signal transducer and activator of transcription 3 (STAT3) activation and cancer cell survival via altered Janus-activated kinase-STAT3 binding. Cancer Res 69: 1958-1965.

Sen B, Peng S, Woods DM, Wistuba I, Bell D, El-Naggar AK, Lai SY, Johnson FM. 2012. STAT5A-mediated SOCS2 expression regulates Jak2 and STAT3 activity following c-Src inhibition in head and neck squamous carcinoma. Clin Cancer Res 18: 127-139.

Sequist LV, Waltman BA, Dias-Santagata D, Digumarthy S, Turke AB, Fidias $\mathrm{P}$, aBergethon $\mathrm{K}$, Shaw AT, Gettinger S, Cosper AK et al. 2011. Genotypic and histological evolution of lung cancers acquiring resistance to EGFR inhibitors. Sci Trans1 Med 3: 75ra26. doi: 10.1126/scitranslmed.3002003.

Serra V, Scaltriti M, Prudkin L, Eichhorn PJ, Ibrahim YH, Chandarlapaty S, Markman B, Rodriguez O, Guzman M, Rodriguez S, et al. 2011. PI3K inhibition results in enhanced HER signaling and acquired ERK dependency in HER2-overexpressing breast cancer. Oncogene 30: 2547-2557. 
Shah OJ, Wang Z, Hunter T. 2004. Inappropriate activation of the TSC/Rheb/mTOR/S6K cassette induces IRS1/2 depletion, insulin resistance, and cell survival deficiencies. Curr Biol 14: 1650-1656.

Shao Y, Aplin AE. 2010. Akt3-mediated resistance to apoptosis in B-RAF-targeted melanoma cells. Cancer Res 70: 66706681.

Sharma SV, Settleman J. 2009. ErbBs in lung cancer. Exp Cell Res 315: 557-571.

Shaul YD, Seger R. 2007. The MEK/ERK cascade: From signaling specificity to diverse functions. Biochim Biophys Acta 1773: 1213-1226.

Shi F, Telesco SE, Liu Y, Radhakrishnan R, Lemmon MA. 2010. ErbB3/HER3 intracellular domain is competent to bind ATP and catalyze autophosphorylation. Proc Natl Acad Sci 107: 7692-7697.

Shi H, Kong X, Ribas A, Lo RS. 2011. Combinatorial treatments that overcome PDGFR $\beta$-driven resistance of melanoma cells to V600EB-RAF inhibition. Cancer Res 71: 5067-5074.

Solit DB, Rosen N. 2011. Resistance to BRAF inhibition in melanomas. $N$ Engl J Med 364: 772-774.

Stein EG, Gustafson TA, Hubbard SR. 2001. The BPS domain of Grb10 inhibits the catalytic activity of the insulin and IGF1 receptors. FEBS Lett 493: 106-111.

Su F, Bradley WD, Wang Q, Yang H, Xu L, Higgins B, Kolinsky K, Packman K, Kim MJ, Trunzer K, et al. 2012a. Resistance to selective BRAF inhibition can be mediated by modest upstream pathway activation. Cancer Res 72: 969978.

Su F, Viros A, Milagre C, Trunzer K, Bollag G, Spleiss O, ReisFilho JS, Kong X, Koya RC, Flaherty KT, et al. 2012b. RAS mutations in cutaneous squamous-cell carcinomas in patients treated with BRAF inhibitors. N Engl J Med 366: 207215.

Tamburini J, Chapuis N, Bardet V, Park S, Sujobert P, Willems L, Ifrah N, Dreyfus F, Mayeux P, Lacombe C, et al. 2008. Mammalian target of rapamycin (mTOR) inhibition activates phosphatidylinositol 3-kinase/Akt by up-regulating insulin-like growth factor-1 receptor signaling in acute myeloid leukemia: Rationale for therapeutic inhibition of both pathways. Blood 111: 379-382.

Treins C, Warne PH, Magnuson MA, Pende M, Downward $\mathrm{J} .2010$. Rictor is a novel target of p70 S6 kinase-1. Oncogene 29: 1003-1016.

Turke AB, Zejnullahu K, Wu YL, Song Y, Dias-Santagata D, Lifshits E, Toschi L, Rogers A, Mok T, Sequist L, et al. 2010. Preexistence and clonal selection of MET amplification in EGFR mutant NSCLC. Cancer Cell 17: 77-88.

Tzivion G, Dobson M, Ramakrishnan G. 2011. FoxO transcription factors; Regulation by AKT and 14-3-3 proteins. Biochim Biophys Acta 1813: 1938-1945.

Villanueva J, Vultur A, Lee JT, Somasundaram R, FukunagaKalabis M, Cipolla AK, Wubbenhorst B, Xu X, Gimotty PA, Kee D, et al. 2010. Acquired resistance to BRAF inhibitors mediated by a RAF kinase switch in melanoma can be overcome by cotargeting MEK and IGF-1R/PI3K. Cancer Cell 18: 683-695.

Wagle N, Emery C, Berger MF, Davis MJ, Sawyer A, Pochanard P, Kehoe SM, Johannessen CM, Macconaill LE, Hahn WC, et al. 2011. Dissecting therapeutic resistance to RAF inhibition in melanoma by tumor genomic profiling. $J$ Clin Oncol 29: 3085-3096.

Wan PT, Garnett MJ, Roe SM, Lee S, Niculescu-Duvaz D, Good VM, Jones CM, Marshall CJ, Springer CJ, Barford D, et al. 2004. Mechanism of activation of the RAF-ERK signaling pathway by oncogenic mutations of B-RAF. Cell 116: 855-867.
Wan X, Harkavy B, Shen N, Grohar P, Helman LJ. 2007. Rapamycin induces feedback activation of Akt signaling through an IGF-1R-dependent mechanism. Oncogene 26: 1932-1940.

Wheeler DL, Iida M, Kruser TJ, Nechrebecki MM, Dunn EF, Armstrong EA, Huang S, Harari PM. 2009. Epidermal growth factor receptor cooperates with Src family kinases in acquired resistance to cetuximab. Cancer Biol Ther 8: 696-703.

Whittaker S, Kirk R, Hayward R, Zambon A, Viros A, Cantarino N, Affolter A, Nourry A, Niculescu-Duvaz D, Springer C et al. 2010. Gatekeeper mutations mediate resistance to BRAF-targeted therapies. Sci Transl Med 2: 35ra41. doi: 10.1126/scitranslmed.3000758.

Wieduwilt MJ, Moasser MM. 2008. The epidermal growth factor receptor family: Biology driving targeted therapeutics. Cell Mol Life Sci 65: 1566-1584.

Xia W, Bacus S, Hegde P, Husain I, Strum J, Liu L, Paulazzo G, Lyass L, Trusk P, Hill J, et al. 2006. A model of acquired autoresistance to a potent ErbB2 tyrosine kinase inhibitor and a therapeutic strategy to prevent its onset in breast cancer. Proc Natl Acad Sci 103: 7795-7800.

Yao Z, Fenoglio S, Gao DC, Camiolo M, Stiles B, Lindsted T, Schlederer M, Johns C, Altorki N, Mittal V, et al. 2010. TGF$\beta$ IL-6 axis mediates selective and adaptive mechanisms of resistance to molecular targeted therapy in lung cancer. Proc Nat1 Acad Sci 107: 15535-15540.

Yonesaka K, Zejnullahu K, Okamoto I, Satoh T, Cappuzzo F, Souglakos J, Ercan D, Rogers A, Roncalli M, Takeda M et al. 2011. Activation of ERBB2 signaling causes resistance to the EGFR-directed therapeutic antibody cetuximab. Sci Transl Med 3: 99ra86. doi: 10.1126/scitranslmed.3002442.

Yu Y, Yoon SO, Poulogiannis G, Yang Q, Ma XM, Villen J, Kubica N, Hoffman GR, Cantley LC, Gygi SP, et al. 2011. Phosphoproteomic analysis identifies Grb10 as an mTORC1 substrate that negatively regulates insulin signaling. Science 332: 1322-1326.

Yuan TL, Cantley LC. 2008. PI3K pathway alterations in cancer: Variations on a theme. Oncogene 27: 5497-5510.

Zhang H, Bajraszewski N, Wu E, Wang H, Moseman AP, Dabora SL, Griffin JD, Kwiatkowski DJ. 2007. PDGFRs are critical for PI3K/Akt activation and negatively regulated by mTOR. J Clin Invest 117: 730-738.

Zhang S, Huang WC, Li P, Guo H, Poh SB, Brady SW, Xiong Y, Tseng LM, Li SH, Ding Z, et al. 2011. Combating trastuzumab resistance by targeting SRC, a common node downstream of multiple resistance pathways. Nat Med 17: 461-469.

Zoncu R, Efeyan A, Sabatini DM. 2011. mTOR: From growth signal integration to cancer, diabetes and ageing. Nat Rev Mol Cell Biol 12: 21-35. 


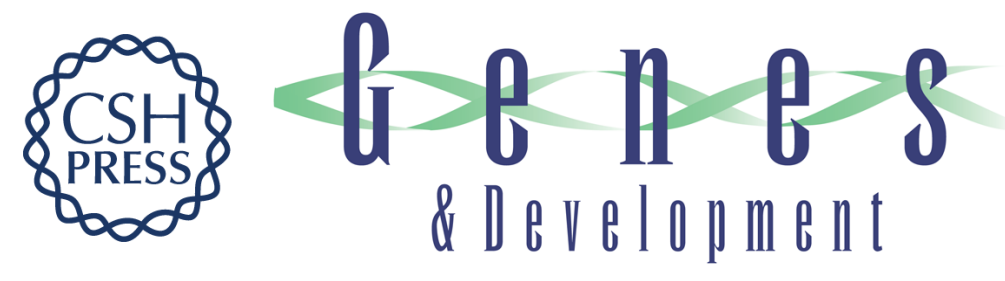

\section{Complexity in the signaling network: insights from the use of targeted inhibitors in cancer therapy}

Jeremy S. Logue and Deborah K. Morrison

Genes Dev. 2012, 26:

Access the most recent version at doi:10.1101/gad.186965.112

References This article cites 80 articles, 33 of which can be accessed free at: http://genesdev.cshlp.org/content/26/7/641.full.html\#ref-list-1

License

Email Alerting Receive free email alerts when new articles cite this article - sign up in the box at the top Service right corner of the article or click here.

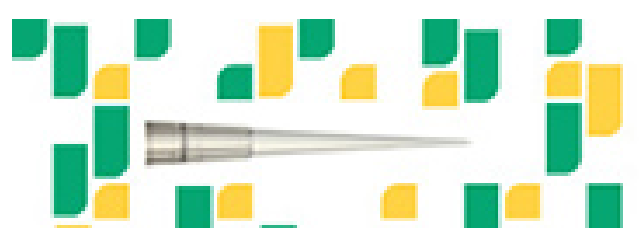

